УДК 517.946

\author{
I. D. Pukal'skit, B. O. Yashan
}

\title{
THE CAUCHY PROBLEM WITH IMPULSE ACTION AND DEGENERATION FOR PARABOLIC EQUATIONS
}

\begin{abstract}
I. D. Pukal'skii, B. O. Yashan. The Cauchy problem with impulse action and degeneration for parabolic equations, Mat. Stud. 52 (2019), 63-70.

We study the Cauchy problem for the second-order linear parabolic equations with impulse conditions in the time variable and power singularity in the coefficients of any order with respect to the time and space variables. By using the maximum principle and apriori estimates we establish the existence and uniqueness of solution of the problem in Hölder spaces with power weight.
\end{abstract}

The study of systems with discontinuous trajectories is stimulated by the development of technology, where impulse computing devices play an important role. Research of tasks in theory of automatic control, theory of nuclear reactors, dynamic systems, leads to solving of boundary value problems for differential equations with impulse action. Fundamental results in the theory of investigation of solutions of systems of ordinary differential equations with impulse action are given in monographs [1-3]. The existence of the periodic solutions of impulse action problems for equations of hyperbolic type has been studied in papers $[4,5]$. In papers [6,7] there are presented results of research on classical solutions of momentum boundary value problems for parabolic degenerate equations. Papers $[8,9]$ are devoted to the boundary value problems with nonlocal and integral conditions on time variable for parabolic equations with degeneration in coefficients on time and space variables.

This article suggests an algorithm for solving of the Cauchy problem for the second-order parabolic equation with degree singularities and degeneracy in coefficients of time and space variables of arbitrary order for some multiple points and impulse actions on time variable at certain points in time. The existence and uniqueness of a solution of the task is proven in Hölder spaces with a degree of weight.

1. Formulation of the Problem and Main Restrictions. Let $\eta, t_{0}, t_{1}, \ldots, t_{N}, t_{N+1}$ be fixed positive numbers, $0 \leq t_{0}<t_{1}<\cdots<t_{N}<t_{N+1}, t_{0}<\eta<t_{N+1}, \eta \neq t_{\lambda}$, $\lambda \in\{1,2, \ldots, N\}$, let $\Omega$ be a some bounded domain, $\bar{\Omega} \subset \mathbb{R}^{n-1}, \operatorname{dim} \Omega \leq n-1$. Let us denote $\Pi_{(0)}=\left(\left\{(t, x) \mid t \in\left[t_{0} ; t_{N+1}\right), x \in \Omega\right\} \cup\left\{(t, x) \mid t=\eta, x \in \mathbb{R}^{n} \backslash \bar{\Omega}\right\}\right)$.

In the domain $\Pi=\left[t_{0}, t_{N+1}\right) \times \mathbb{R}^{n}$ we consider the problem of finding the function $u(t, x)$ which for $t \neq t_{\lambda},(t, x) \notin \Pi_{(0)}$ satisfies the equation

$$
(L u)(t, x)=\left[\partial_{t}-\sum_{i j=1}^{n} A_{i j}(t, x) \partial_{x_{i}} \partial_{x_{j}}+\sum_{i=1}^{n} A_{i}(t, x) \partial_{x_{i}}+A_{0}(t, x)\right] u(t, x)=f(t, x),
$$

2010 Mathematics Subject Classification: 35K65, 35K67.

Keywords: degeneration; impulse action; a priori estimation; boundary condition. doi: $10.30970 / \mathrm{ms} .52 .1 .63-70$

(C) I. D. Pukal'skii, B. O. Yashan, 2019 
and conditions for the time variable

$$
\begin{gathered}
u\left(t_{0}+0, x\right)=\varphi_{0}(x) \\
u\left(t_{\lambda}+0, x\right)-u\left(t_{\lambda}-0, x\right)=b_{\lambda}(x) u\left(t_{\lambda}-0, x\right)+\varphi_{\lambda}(x) .
\end{gathered}
$$

The power singularities of coefficients of differential equation (1) at the point $P(t, x) \in$ $\Pi \backslash \Pi_{(0)}$ are characterized by the functions $s_{1}\left(\beta_{i}^{(1)}, t\right)$ and $s_{2}\left(\beta_{i}^{(2)}, x\right): s_{1}\left(\beta_{i}^{(1)}, t\right)=|t-\eta|^{\beta_{i}^{(1)}}$ as $|t-\eta| \leq 1, s_{1}\left(\beta_{i}^{(1)}, t\right) \equiv 1$ as $|t-\eta| \geq 1 ; s_{2}\left(\beta_{i}^{(2)}, x\right)=\rho^{\beta_{i}^{(2)}}(x)$ as $\rho(x) \leq 1, s_{2}\left(\beta_{i}^{(2)}, x\right)=1$ as $\rho(x) \geq 1, \rho(x)=\inf _{z \in \bar{\Omega}}|x-z|, \beta_{i}^{(\nu)} \in(-\infty, \infty), \nu \in\{1,2\}, \beta^{(\nu)}=\left(\beta_{1}^{(\nu)}, \beta_{2}^{(\nu)}, \ldots, \beta_{n}^{(\nu)}\right)$, $\beta=\left(\beta^{(1)}, \beta^{(2)}\right)$.

Denote by $\left(x_{1}^{(1)}, \ldots, x_{i}^{(1)}, \ldots, x_{n}^{(1)}\right)$ the coordinates of the point $x^{(1)} \in \mathbb{R}^{n}$. Let $\left(x_{1}^{(2)}, \ldots\right.$, $\left.x_{i}^{(2)}, \ldots, x_{n}^{(2)}\right)$ be the coordinates of the point $x^{(2)} \in \mathbb{R}^{n}, l, \alpha, q^{(1)}, q^{(2)}, \gamma^{(1)}, \gamma^{(2)}, \mu_{j}^{(1)}, \mu_{j}^{(2)}$ be real numbers, $j \in\{0,1, \ldots, n\}, q^{(\nu)} \geq 0, \gamma^{(\nu)} \geq 0, l \geq 0, \mu_{j}^{(\nu)} \geq 0, \alpha \in(0,1)$, [l] be the integer part of $l$. Let $D$ be an arbitrary closed domain, $\bar{D} \subset \mathbb{R}^{n}, Q^{(k)}=\left[t_{k}, t_{k+1}\right) \times D$, $P_{1}^{(k)}\left(t^{(1)}, x^{(1)}\right), R^{(k)}\left(t^{(2)}, x^{(2)}\right), H^{(k)}\left(t^{(1)}, x^{(2)}\right)$ be arbitrary points of the domain $Q^{(k)}, \bar{Q}^{(k)} \subset$ $\Pi^{(k)}=\left[t_{k}, t_{k+1}\right) \times \mathbb{R}^{n}, k \in\{0,1, \ldots, N\}, Q_{(0)}=Q^{(k)} \bigcap \Pi_{(0)}$.

Below we define the function space to study problem (1)-(3).

Let $H^{l}(\gamma ; \beta ; q ; \Pi)$ be the set of functions $u(t, x) \in L_{1}(\Pi)$ having continuous partial derivatives in $Q^{(k)} \backslash Q_{(0)}$ of the form $\partial_{t}^{j} \partial_{x}^{r} u, 2 i+|r| \leq[l]$ and a finite value of the norm

$$
\|u ; \gamma ; \beta ; q ; \Pi\|_{l}=\sup _{k}\left\{\sum_{2 j+|r| \leq[l]}\left\|u ; \gamma ; \beta ; q, Q^{(k)}\right\|_{2 j+|r|}+\left\langle u ; \gamma ; \beta ; q ; Q^{(k)}\right\rangle_{l}\right\},
$$

where, e.g.,

$$
\begin{gathered}
\left\|u ; \gamma ; \beta ; 0 ; Q^{(k)}\right\|_{0}=\sup _{k}\left(\sup _{P_{1}^{(k)} \in \bar{Q}^{(k)}}\left|u\left(P_{1}^{(k)}\right)\right|\right) \equiv \sup _{k}\left\|u ; Q^{(k)}\right\|_{0}, \\
\left\|u ; \gamma ; \beta ; q ; Q^{(k)}\right\|_{2 j+|r|}=\sup _{P_{1}^{(k)} \in \bar{Q}^{(k)}}\left[s_{1}\left(q^{(1)}+2 j \gamma^{(1)}, t^{(1)}\right) s_{2}\left(q^{(2)}+2 j \gamma^{(2)}, x^{(1)}\right) \times\right. \\
\left.\times \prod_{i=1}^{n} s_{1}\left(r_{i}\left(\gamma^{(1)}-\beta_{i}^{(1)}\right), t^{(1)}\right) s_{2}\left(r_{i}\left(\gamma^{(2)}-\beta_{i}^{(2)}\right), x^{(1)}\right)\left|\partial_{t}^{j} \partial_{x}^{r} u\left(P_{1}^{(k)}\right)\right|\right], \\
\left\langle u ; \gamma ; \beta ; q ; Q^{(k)}\right\rangle_{l}=\sum_{2 j+|r|=[l]}\left\{\sum _ { \nu = 1 } ^ { n } \operatorname { s u p } _ { ( P _ { 1 } ^ { ( k ) } , H ^ { ( k ) } ) \subset \overline { Q } ^ { ( k ) } } \left[s_{1}\left(q^{(1)}+l \gamma^{(1)}, t^{(1)}\right) \times\right.\right. \\
\times s_{2}\left(q^{(2)}+2 j \gamma^{(2)}, \tilde{x}\right) \prod_{i=1}^{n} s_{1}\left(-r_{i} \beta_{i}^{(1)}, t^{(1)}\right) s_{2}\left(r_{i}\left(\gamma^{(2)}-\beta_{i}^{(2)}, \tilde{x}\right)\right) s_{1}\left(-\{l\} \beta_{\nu}^{(1)}, t^{(1)}\right) \times \\
\times s_{2}\left(\{l\}\left(\gamma^{(2)}-\beta_{\nu}^{(2)}, \tilde{x}\right)\left|x_{\nu}^{(1)}-x_{\nu}^{(2)}\right|^{-\{l\}} \mid \partial_{t}^{j} \partial_{x}^{r} u\left(P_{1}^{(k)}\right)-\right. \\
\left.-\partial_{t}^{j} \partial_{x}^{r} u\left(H^{(k)}\right) \mid\right]+\sup _{\left(R^{(k)}, H^{(k)}\right) \subset \bar{Q}^{(k)}}\left[s _ { 1 } ( q ^ { ( 1 ) } + l \gamma ^ { ( 1 ) } , \tilde { t } ) s _ { 2 } \left(q^{(2)}+\left(l-|r| \gamma^{(2)}, x^{(2)}\right) \times\right.\right. \\
\left.\left.\times \prod_{i=1}^{n} s_{1}\left(-r_{i} \beta_{i}^{(1)}, \tilde{t}\right) s_{2}\left(r_{i}\left(\gamma^{(2)}-\beta_{i}^{(2)}, x^{(2)}\right)\right)\left|t^{(1)}-t^{(2)}\right|^{-\left\{\frac{l}{2}\right\}}\left|\partial_{t}^{j} \partial_{x}^{r} u\left(R^{(k)}\right)-\partial_{t}^{j} \partial_{x}^{r} u\left(H^{(k)}\right)\right|\right]\right\} .
\end{gathered}
$$

Here: $s_{1}(a, \tilde{t})=\min \left(s_{1}\left(a, t^{(1)}\right), s_{1}\left(a, t^{(2)}\right)\right), \partial_{x}^{r}=\partial_{x_{1}}^{r_{1}} \partial_{x_{2}}^{r_{2}} \ldots \partial_{x_{n}}^{r_{n}},|r|=r_{1}+r_{2}+\cdots+r_{n}$, $s_{2}(a, \tilde{x})=\min \left(s_{2}\left(a, x^{(1)}\right), s_{2}\left(a, x^{(2)}\right)\right), l=[l]+\{l\}$. 
Assume that initial problem (1)-(3) satisfies the following conditions:

a) for an arbitrary vector $\xi=\left(\xi_{1}, \xi_{2}, \ldots, \xi_{n}\right), \forall(t, x) \in \Pi \backslash \Pi_{(0)}$ the following inequality holds

$$
\pi_{1}|\xi|^{2} \leq \sum_{i j=1}^{n} s_{1}\left(\beta_{i}^{(1)}, t\right) s_{1}\left(\beta_{j}^{(1)}, t\right) s_{2}\left(\beta_{i}^{(2)}, x\right) s_{2}\left(\beta_{j}^{(2)}, x\right) A_{i j}(t, x) \xi_{i} \xi_{j} \leq \pi_{2}|\xi|^{2}
$$

where $\pi_{1}, \pi_{2}$ are fixed positive constants and $s_{1}\left(\mu_{i}^{(1)}, t\right) s_{2}\left(\mu_{i}^{(2)}, x\right) A_{i} \in H^{\alpha}\left(\gamma ; \beta ; 0 ; \Pi \backslash \Pi_{(0)}\right)$,

$$
\begin{gathered}
s_{1}\left(\beta_{i}^{(1)}, t\right) s_{1}\left(\beta_{j}^{(1)}, t\right) s_{2}\left(\beta_{i}^{(2)}, x\right) s_{2}\left(\beta_{j}^{(2)}, x\right) A_{i j} \in H^{\alpha}\left(\gamma ; \beta ; 0 ; \Pi \backslash \Pi_{(0)}\right), \\
s_{1}\left(\mu_{0}^{(1)}, t\right) s_{2}\left(\mu_{0}^{(2)}, x\right) A_{0} \in H^{\alpha}\left(\gamma ; \beta ; 0 ; \Pi \backslash \Pi_{(0)}\right), A_{0} \geq-a, a \geq 0, \\
\gamma^{(\nu)}=\max \left\{\max _{i}\left(1+\beta_{i}^{(\nu)}\right), \max _{i}\left(\mu_{i}^{(\nu)}-\beta_{i}^{(\nu)}\right), \frac{\mu_{0}^{(\nu)}}{2}\right\}, \nu \in\{1,2\} ;
\end{gathered}
$$

b) functions $f \in H^{\alpha}\left(\gamma ; \beta ; \mu_{0} ; \Pi \backslash \Pi_{(0)}\right), \varphi_{0} \in H^{2+\alpha}\left(\tilde{\gamma} ; \tilde{\beta} ; 0 ; \mathbb{R}^{n} \backslash \bar{\Omega}\right), \varphi_{\lambda} \in H^{2+\alpha}(\tilde{\gamma} ; \tilde{\beta} ; 0 ;(\Pi \backslash$ $\left.\Pi_{(0)} \mid \cap\left(t=t_{\lambda}\right)\right), b_{\lambda}(x) \in C^{2+\alpha}\left(\left(\Pi \backslash \Pi_{(0)}\right) \cap\left(t=t_{\lambda}\right)\right), \tilde{\gamma}=\left(0, \gamma^{(2)}\right), \tilde{\beta}=\left(0, \beta^{(2)}\right)$.

Let us formulate the main result of the paper.

Theorem 1. Let conditions a), b) be satisfied for problem (1)-(3). Then there exists the unique solution of problem (1)-(3) in the space $H^{2+\alpha}\left(\gamma, \beta ; 0 ; \Pi \backslash \Pi_{(0)}\right)$ and the following estimate is correct:

$$
\begin{aligned}
& \left\|u ; \gamma ; \beta ; 0 ; \Pi \backslash \Pi_{(0)}\right\|_{2+\alpha} \leq c\left\{\sum_{k=1}^{N} \prod_{\lambda=k}^{N}\left(1+\left\|b_{\lambda} ; \Pi \cap\left(t=t_{\lambda}\right)\right\|_{0}\right) \times\right. \\
& \times\left(\left\|\varphi_{k-1} ; \tilde{\gamma} ; \tilde{\beta} ; 0 ; \Pi \cap\left(t=t_{k-1}\right)\right\|_{2+\alpha}+\left\|f ; \gamma ; \beta ; \mu_{0} ; \Pi^{(k-1)}\right\|_{\alpha}\right)+ \\
& \left.+\left\|\varphi_{N} ; \tilde{\gamma} ; \tilde{\beta} ; 0 ; \Pi \cap\left(t=t_{N}\right)\right\|_{2+\alpha}+\left\|f ; \gamma ; \beta ; \mu_{0} ; \Pi^{(N)}\right\|_{\alpha}\right\} \equiv B .
\end{aligned}
$$

To prove Theorem 1 we construct a sequence of solutions of problems with smooth coefficients, for which the boundary value is the solution of problem (1)-(3).

2. Evaluation of solutions of problems with smooth coefficients. Let $\Pi_{m}=\Pi \cap$ $\left\{(t, x) \in \Pi \mid s_{1}(1, t) \geq m_{1}^{-1}, s_{2}(1, x) \geq m_{2}^{-1}\right\}, m_{1}>1, m_{2}>1$ be a sequence of domains converging to $\Pi \backslash \Pi_{(0)}$ as $m_{1} \rightarrow \infty, m_{2} \rightarrow \infty$.

In the domain $\Pi$ we consider the problem of finding the function $u_{m}(t, x)$, that satisfies the equation

$$
\left(L_{1} u_{m}\right)(t, x) \equiv\left(\partial_{t}-\sum_{i j=1}^{n} a_{i j}(t, x) \partial_{x_{i}} \partial_{x_{j}}+\sum_{i=1}^{n} a_{i}(t, x) \partial_{x_{i}}+a_{0}(t, x)\right) u_{m}(t, x)=f_{m}(t, x)
$$

and conditions concerning to the time variable

$$
\begin{gathered}
u_{m}\left(t_{0}+0, x\right)=\varphi_{0}^{(m)}(x) \\
u_{m}\left(t_{\lambda}+0, x\right)-u_{m}\left(t_{\lambda}-0, x\right)=b_{\lambda}^{(m)}(x) u_{m}\left(t_{\lambda}-0, x\right)+\varphi_{\lambda}^{(m)}(x) .
\end{gathered}
$$

Here the coefficients $a_{i j}, a_{i}, a_{0}, b_{\lambda}^{(m)}(x)$ and functions $f_{m}, \varphi_{0}^{(m)}, \varphi_{\lambda}^{(m)}$ for $(t, x) \in \Pi_{m}$ coincide with $A_{i j}, A_{i}, A_{0}, b_{\lambda}$ and $f, \varphi_{0}, \varphi_{\lambda}$, respectively. For $(t, x) \in \Pi \backslash \Pi_{m}$ the coefficients $a_{i j}, a_{i}, a_{0}, b_{\lambda}^{(m)}$, and functions $f_{m}, \varphi_{0}^{(m)}, \varphi_{\lambda}^{(m)}$ are continuous extensions of the coefficients 
$A_{i j}, A_{i}, A_{0}, b_{\lambda}$ and functions $f, \varphi_{0}, \varphi_{\lambda}$ from the domain $\Pi_{m}$ into the domain $\Pi \backslash \Pi_{m}[10$, p. 83], $m=\left(m_{1}, m_{2}\right)$.

Let us find the estimate of the solution of problem (5)-(7). In the space $C^{2+\alpha}(\Pi)$ we introduce the norm $\left\|u_{m} ; \gamma ; \beta ; q ; \Pi\right\|_{l}$, which for every $m$ is equivalent to the Hölder norm determined by analogy as $\|u ; \gamma ; \beta ; q ; \Pi\|_{l}$. But instead of the functions $s_{1}\left(\beta_{i}^{(1)}, t\right), s_{2}\left(\beta_{i}^{(2)}, x\right)$ we take $d_{1}\left(\beta_{i}^{(1)}, t\right), d_{2}\left(\beta_{i}^{(2)}, x\right): d_{1}\left(\beta_{i}^{(1)}, t\right)=\max \left(s_{1}\left(\beta_{i}^{(1)}, t\right), m_{1}^{-\beta_{i}^{(1)}}\right)$ as $\beta_{i}^{(1)} \geq 0$ and $d_{1}\left(\beta_{i}^{(1)}, t\right)=$ $\min \left(s_{1}\left(\beta_{i}^{(1)}, t\right), m_{1}^{-\beta_{i}^{(1)}}\right)$ as $\beta_{i}^{(1)}<0 ; d_{2}\left(\beta_{i}^{(2)}, x\right)=\max \left(s_{2}\left(\beta_{i}^{(2)}, x\right), m_{2}^{-\beta_{i}^{(2)}}\right)$ as $\beta_{i}^{(2)} \geq 0$ and $d_{2}\left(\beta_{i}^{(2)}, x\right)=\min \left(s_{2}\left(\beta_{i}^{(2)}, x\right), m_{2}^{-\beta_{i}^{(2)}}\right)$ as $\beta_{i}^{(2)}<0$.

To solve problem (5)-(7), we have a valid theorem.

Theorem 2. Let $u_{m}$ be the classical solutions of problem (5)-(7) in the domain $\Pi$ and let conditions a), b) be satisfied. Then for $u_{m}(t, x)$ the estimate is true

$$
\begin{gathered}
\left|u_{m}\right| \leq \sum_{k=1}^{N} \prod_{\lambda=k}^{N}\left(1+\left\|b_{\lambda}^{(m)} ; \Pi \cap\left(t=t_{\lambda}\right)\right\|_{0}\right)\left(\left\|\varphi_{(k-1)}^{(m)} ; \Pi \cap\left(t=t_{k-1}\right)\right\|_{0}+\right. \\
\left.+t_{k}\left\|f_{m} ; \Pi^{(k-1)}\right\|_{0}\right) e^{a t_{k}}+\left(\left\|\varphi_{N}^{(m)} ; \Pi \cap\left(t=t_{N}\right)\right\|_{0}+t_{N+1}\left\|f_{m} ; \Pi^{(N)}\right\|_{0}\right) e^{a t_{N+1}} .
\end{gathered}
$$

Proof. Inequality (8) can be proved by the scheme of proving in Theorem 2.5 from [11, p. 27 . In view of this theorem for the solution of problem (5)-(6) in $\Pi^{(0)}$ we estimate

$$
\left\|u_{m} ; \Pi^{(0)}\right\|_{0} \leq\left(\left\|\varphi_{0}^{(m)} ; \Pi^{(0)} \cap\left(t=t_{0}\right)\right\|_{0}+t_{1}\left\|f_{m} ; \Pi^{(0)}\right\|_{0}\right) e^{a t_{1}} .
$$

If $k \geq 1$, then from given inequality (7) we obtain inequalities

$$
\begin{gathered}
\left\|u_{m} ; \Pi^{(k)}\right\|_{0} \leq\left[( 1 + \| b _ { k } ^ { ( m ) } ; \Pi ^ { ( k ) } \cap ( t = t _ { k } ) \| _ { 0 } ) \left(\left\|u_{m} ; \Pi^{(k-1)}\right\|_{0}+\right.\right. \\
\left.\left.+\left\|\varphi_{k}^{(m)} ; \Pi^{(k)} \cap\left(t=t_{k}\right)\right\|_{0}+t_{k+1}\left\|f_{m} ; \Pi^{(k)}\right\|_{0}\right)\right] e^{a t_{k+1}}, \quad k \in\{1,2, \ldots, N\} .
\end{gathered}
$$

Combining inequalities (9), (10) we obtain estimate (8).

Theorem 3. If conditions a), b) are satisfied, then the inequality solving problem (5)-(7) is

$$
\begin{aligned}
& \left\|u_{m} ; \gamma ; \beta ; 0 ; \Pi\right\|_{2+\alpha} \leq c\left\{\sum _ { k = 1 } ^ { N } \left[\prod_{\lambda=k}^{N}\left(1+\left\|b_{\lambda}^{(m)} ; \Pi \cap\left(t=t_{\lambda}\right)\right\|_{0}\right) \times\right.\right. \\
& \times\left(\left\|\varphi_{k-1}^{(m)} ; \tilde{\gamma} ; \tilde{\beta} ; 0 ; \Pi \cap\left(t=t_{k-1}\right)\right\|_{2+\alpha}+\left\|f_{m} ; \gamma ; \beta ; \mu_{0} ; \Pi^{(k-1)}\right\|_{\alpha}\right)+ \\
& \left.\left.\quad+\left\|\varphi_{N}^{(m)} ; \tilde{\gamma} ; \tilde{\beta} ; 0 ; \Pi \cap\left(t=t_{N}\right)\right\|_{2+\alpha}+\left\|f_{m} ; \gamma ; \beta ; \mu_{0} ; \Pi^{(N)}\right\|_{\alpha}\right]\right\} .
\end{aligned}
$$

Proof. Using the definition of the norm and interpolation inequalities from [12 p. 38], we have

$$
\left\|u_{m} ; \gamma ; \beta ; 0 ; \Pi^{(k)}\right\|_{2+\alpha} \leq\left(1+\varepsilon^{\alpha}\right)\left\langle u_{m} ; \gamma ; \beta ; 0 ; \Pi^{(k)}\right\rangle_{2+\alpha}+c(\varepsilon)\left\|u_{m} ; \Pi^{(k)}\right\|_{0},
$$

where $\varepsilon$ is an arbitrary real number, $\varepsilon \in(0,1)$. It is sufficient to evaluate the half norm $\left\langle u_{m} ; \gamma ; \beta ; 0 ; \Pi^{(k)}\right\rangle_{2+\alpha}$. From the definition of the half norm it follows that there exist the points $P_{1}^{(k)}, R^{(k)}, H^{(k)}$ in $\Pi^{(k)}$ for which one of the following inequalities holds

$$
\frac{1}{2}\left\|u_{m} ; \gamma ; \beta ; 0 ; \Pi^{(k)}\right\|_{2+\alpha} \leq E_{\delta}, \quad \delta \in\{1,2\},
$$


where

$$
\begin{gathered}
E_{1}=\sum_{2 j+|r|=2} \sum_{\nu=1}^{n} d_{1}\left((2+\alpha) \gamma^{(1)}, t^{(1)}\right) \prod_{i=1}^{n} d_{1}\left(-r_{i} \beta_{i}^{(1)}, t^{(1)}\right) d_{2}\left(r_{i}\left(\gamma^{(2)}-\beta_{i}^{(2)}\right), \tilde{x}\right) \times \\
\times d_{1}\left(-\alpha \beta_{\nu}^{(1)}, t^{(1)}\right) d_{2}\left(-\alpha\left(\gamma^{(2)}-\beta_{\nu}^{(2)}, \tilde{x}\right)\left|x_{\nu}^{(1)}-x_{\nu}^{(2)}\right|^{-\alpha}\left|\partial_{t}^{j} \partial_{x}^{r} u\left(P_{1}^{(k)}\right)-\partial_{t}^{j} \partial_{x}^{r} u\left(H^{(k)}\right)\right|,\right. \\
E_{2}=\sum_{2 j+|r|=2} d_{1}\left((2+\alpha) \gamma^{(1)} ; \tilde{t}\right) d_{2}\left((2-|r|+\alpha) \gamma^{(2)} ; x^{(2)}\right) \times \\
\times \prod_{i=1}^{n} d_{1}\left(-r_{i} \beta_{i}^{(1)}, \tilde{t}\right) d_{2}\left(r_{i}\left(\gamma^{(2)}-\beta_{i}^{(2)}, x^{(2)}\right)\left|t^{(1)}-t^{(2)}\right|^{-\frac{\alpha}{2}}\left|\partial_{t}^{j} \partial_{x}^{r} u\left(R^{(k)}\right)-\partial_{t}^{j} \partial_{x}^{r} u\left(H^{(k)}\right)\right|,\right.
\end{gathered}
$$

$d_{1}(a, \tilde{t})=\min \left(d_{1}\left(a, t^{(1)}\right), d_{1}\left(a, t^{(2)}\right)\right), d_{2}(a, \tilde{x})=\min \left(d_{2}\left(a, x^{(1)}\right), d_{2}\left(a, x^{(2)}\right)\right)$.

If $\left|x_{\nu}^{(1)}-x_{\nu}^{(2)}\right| \geq n^{-1} d_{1}\left(\gamma^{(1)}-\beta_{\nu}^{(1)}, t^{(1)}\right) d_{2}\left(\gamma^{(2)}-\beta_{\nu}^{(2)}, \tilde{x}\right) \frac{\varepsilon_{1}}{4} \equiv T_{1}$, then

$$
E_{1} \leq 2 \varepsilon_{1}^{-\alpha}\left\|u_{m} ; \gamma ; \beta ; 0 ; \Pi^{(k)}\right\|_{2} \text {. }
$$

If $\left|t^{(1)}-t^{(2)}\right| \geq d_{1}\left(2 \gamma^{(1)}, \tilde{t}\right) d_{2}\left(2 \gamma^{(2)}, x^{(2)}\right) \frac{\varepsilon_{1}}{16} \equiv T_{2}$, then

$$
E_{2} \leq 2 \varepsilon_{1}^{-\alpha}\left\|u_{m} ; \gamma ; \beta ; 0 ; \Pi^{(k)}\right\|_{2}
$$

Applying the interpolation inequalities to (13), (14), we obtain

$$
E_{\delta} \leq \varepsilon^{\alpha}\left\|u_{m} ; \gamma ; \beta ; 0 ; \Pi^{(k)}\right\|_{2+\alpha}+c(\varepsilon)\left\|u_{m} ; \Pi^{(k)}\right\|_{0} .
$$

Let $\left|x_{\nu}^{(1)}-x_{\nu}^{(2)}\right| \leq T_{1}$ and $\left|t^{(1)}-t^{(2)}\right| \leq T_{2}$. Assume that

$$
d_{1}\left(2 \gamma^{(1)}, \tilde{\tau}\right)=d_{1}\left(2 \gamma^{(1)}, t^{(1)}\right),
$$

$d_{2}\left(2 \gamma^{(2)}, \tilde{x}\right)=d_{2}\left(2 \gamma^{(2)}, x^{(2)}\right),\left(t^{(1)}, x^{(2)}\right) \in \Pi^{(k)}$. In the domain $\Pi^{(k)}$ we write problem $(5)-(7)$ in the form

$$
\begin{gathered}
\left(L_{2} u_{m}\right) \equiv\left(\partial_{t}-\sum_{i j=1}^{n} a_{i j}\left(t^{(1)}, x^{(2)}\right) \partial_{x_{i}} \partial_{x_{j}}\right) u_{m}=\sum_{i j=1}^{n}\left[a_{i j}(t, x)-a_{i j}\left(t^{(1)}, x^{(2)}\right)\right] \partial_{x_{i}} \partial_{x_{j}} u_{m}- \\
-\sum_{i=1}^{n} a_{i}(t, x) \partial_{x_{i}} u_{m}-a_{0}(t, x) u_{m}+f_{m}(t, x) \equiv F_{m}\left(t, x ; u_{m}\right)+f_{m}(t, x), \\
u_{m}\left(t_{k}+0, x\right)=\psi_{m}^{(k)}\left(t_{k}, x\right),
\end{gathered}
$$

where $\psi_{m}^{(0)}\left(t_{0}, x\right)=\varphi_{0}^{(m)}(x), x \in \mathbb{R}^{n}$;

$$
\psi_{m}^{(k)}\left(t_{k}, x\right)=\left[1+b_{k}^{(m)}(x)\right] u_{m}\left(t_{k}-0, x\right)+\varphi_{k}^{(m)}(x), x \in \Pi^{(k)} \cap\left(t=t_{k}\right), \quad k \in\{1,2, \ldots, N\} .
$$

Let $V_{\varepsilon_{2}}$ be a domain from $\Pi^{(k)}, V_{\varepsilon_{2}}=\left\{(t, x) \in \Pi^{(k)},\left|t-t^{(1)}\right| \leq \varepsilon_{2}^{2} T_{2},\left|x_{i}-x_{i}^{(2)}\right| \leq \varepsilon_{2} T_{1}\right.$, $i \in\{1, \ldots, n\}\}$. In problem (16), (17) we make the substitution $u_{m}(t, x)=v_{m}(t, y), y_{i}=$ $d_{1}\left(\beta_{i}^{(1)}, t^{(1)}\right) d_{2}\left(\beta_{i}^{(2)}, x^{(2)}\right) x_{i}$. As a result, we deduce

$$
\left(L_{2} v_{m}\right)(t, y)=\left[\partial_{t}-\sum_{i j=1}^{n} a_{i j}\left(t^{(1)}, x^{(2)}\right) d_{1}\left(\beta_{i}^{(1)}, t^{(1)}\right) d_{1}\left(\beta_{j}^{(1)}, t^{(1)}\right) \times\right.
$$




$$
\begin{gathered}
\left.\times d_{2}\left(\beta_{i}^{(2)}, x^{(2)}\right) d_{2}\left(\beta_{j}^{(2)}, x^{(2)}\right) \partial_{y_{i}} \partial_{y_{j}}\right] v_{m}=F_{m}\left(t, \tilde{y} ; v_{m}\right)+f_{m}(t, \tilde{y}), \\
v_{m}\left(t_{k}+0, y\right)=\psi_{m}^{(k)}\left(t_{k}, \tilde{y}\right)
\end{gathered}
$$

where $\tilde{y}=\left(d_{1}\left(-\beta_{1}^{(1)}, t^{(1)}\right) d_{2}\left(-\beta_{1}^{(2)}, x^{(2)}\right) y_{1}, \ldots, d_{1}\left(-\beta_{n}^{(1)}, t^{(1)}\right) d_{2}\left(-\beta_{n}^{(2)}, x^{(2)}\right) y_{n}\right)$.

Denote $y_{i}^{(2)}=d_{1}\left(\beta_{i}^{(1)}, t^{(1)}\right) d_{2}\left(\beta_{i}^{(2)}, x^{(2)}\right) x_{i}^{(2)}, W_{\varepsilon_{2}}^{(k)}=\left\{(t, y),\left|t-t^{(1)}\right| \leq \varepsilon_{2}^{2} T_{2},\left|y_{i}-y_{i}^{(2)}\right| \leq\right.$ $\left.\varepsilon_{2} T_{2}^{1 / 2}\right\}$ and take the triply differentiable function $\mu(t, y)$, that satisfies the conditions

$$
\mu(t, y)= \begin{cases}1, & (t, y) \in W_{1 / 2}^{(k)}, 0 \leq \mu(t, y) \leq 1 \\ 0, & (t, y) \notin W_{3 / 4}^{(k)},\left|\partial_{t}^{j} \partial_{x}^{r} \mu\right| \leq \\ & \leq c_{j r} d_{1}\left(-2 j \gamma^{(1)} ; t^{(1)}\right) \prod_{i=1}^{n} d_{2}\left(-r_{i} \gamma^{(2)}, x^{(2)}\right) .\end{cases}
$$

In view of (18), (19), the function $Z_{m}(t, y)=v_{m}(t, y) \mu(t, y)$ is a solution of the Cauchy problem

$$
\begin{gathered}
\left(L_{2} Z_{m}\right)(t, y)=\sum_{i j=1}^{n} a_{i j}\left(t^{(1)}, x^{(2)}\right) d_{1}\left(\beta_{i}^{(1)}, t^{(1)}\right) d_{1}\left(\beta_{j}^{(1)}, t^{(1)}\right) d_{2}\left(\beta_{i}^{(2)}, x^{(2)}\right) d_{2}\left(\beta_{j}^{(2)}, x^{(2)}\right) \times \\
\times\left[\partial_{y_{i}} \mu \partial_{y_{j}} v_{m}+\partial_{y_{j}} \mu \partial_{y_{i}} v_{m}\right]+v_{m}\left[\sum_{i j=1}^{n} a_{i j}\left(t^{(1)}, x^{(2)}\right) d_{1}\left(\beta_{i}^{(1)}, t^{(1)}\right) d_{1}\left(\beta_{j}^{(1)}, t^{(1)}\right) \times\right. \\
\left.\times d_{2}\left(\beta_{j}^{(2)}, t^{(2)}\right) d_{2}\left(\beta_{j}^{(2)}, x^{(2)}\right) \partial_{y_{i}} \partial_{y_{j}} \mu-\partial_{t} \mu\right]+\mu\left[F_{m}+f_{m}\right] \equiv F_{m}^{(1)}+\mu f_{m} \\
Z_{m}\left(t_{k}+0, x\right)=\psi_{m}^{(k)}\left(t_{k}, \tilde{y}\right) \mu\left(t_{k}, y\right) .
\end{gathered}
$$

By Theorem 5.1 from [11, p. 364], for the solution of problem (20), (21) inequalities hold

$$
\begin{gathered}
\left|y^{(1)}-y^{(2)}\right|^{-\alpha}\left|\partial_{t}^{j} \partial_{y}^{r} Z_{m}\left(t, y^{(1)}\right)-\partial_{t}^{j} \partial_{y}^{r} Z_{m}\left(t, y^{(2)}\right)\right| \leq c\left(\left\|F_{m}^{(1)}+\mu F_{m}\right\|_{C^{\alpha}\left(W_{3 / 4}^{(k)}\right)}+\right. \\
\left.+\left\|\mu \psi_{m}^{(k)}\right\|_{C^{2+\alpha}\left(W_{3 / 4}^{(k)} \cap\left(t=t_{k}\right)\right)}\right) \equiv B_{m} \\
\left|t^{(1)}-t^{(2)}\right|^{-\alpha / 2}\left|\partial_{t}^{j} \partial_{y}^{r} Z_{m}\left(t^{(1)}, y\right)-\partial_{t}^{j} \partial_{y}^{r} Z_{m}\left(t^{(2)}, y\right)\right| \leq c_{1} B_{m}
\end{gathered}
$$

where $\left\{\left(t, y^{(1)}\right),\left(t, y^{(2)}\right),\left(t^{(1)}, y\right),\left(t^{2}, y\right)\right\} \subset W_{1 / 4}^{(k)}, 2 j+|r|=2$.

Taking into account the properties of the function $\mu(t, y)$, we find

$$
\begin{gathered}
\left\|F_{m}^{(1)}+\mu f_{m}\right\|_{C^{\alpha}\left(W_{3 / 4}^{(k)}\right)} \leq \\
\leq c_{2} d_{1}\left(-(2+\alpha) \gamma^{(1)} ; t^{(1)}\right) d_{2}\left(-(2+\alpha) \gamma^{(2)} ; x^{(2)}\right)\left(\left\|v_{m} ; \gamma ; 0 ; 0 ; W_{3 / 4}^{(k)}\right\|_{2}+\right. \\
\left.+\left\|v_{m} ; W_{3 / 4}^{(k)}\right\|_{0}+\left\|f_{m} ; \gamma ; 0 ; 2 \gamma ; W_{3 / 4}^{(k)}\right\|_{\alpha}+\left\|F_{m} ; \gamma ; 0 ; 2 \gamma ; W_{3 / 4}^{(1)}\right\|_{\alpha}\right), \\
\left\|\mu \psi_{m}^{(k)}\right\|_{C^{2+\alpha}\left(W_{3 / 4}^{(k)} \cap\left(t=t_{k}\right)\right)} \leq c_{3} d_{1}\left(-(2+\alpha) \gamma^{(1)}, t^{(1)}\right) d_{2}\left(-(2+\alpha) \gamma^{(2)}, x^{(2)}\right) \times \\
\times\left\|\psi_{m}^{(k)} ; \tilde{\gamma} ; 0 ; 0 ; W_{3 / 4}^{(k)} \cap\left(t=t_{k}\right)\right\|_{2+\alpha},
\end{gathered}
$$

Substituting (24), (25) into (22), (23) and returning to the variables $(t, x)$, we obtain

$$
\begin{gathered}
E_{\delta} \leq c_{4}\left(\left\|F_{m} ; \gamma ; \beta ; 2 \gamma ; V_{3 / 4}^{(k)}\right\|_{\alpha}+\left\|f_{m} ; \gamma ; \beta ; 2 \gamma ; V_{3 / 4}^{(k)}\right\|_{\alpha}+\right. \\
\left.+\left\|u_{m} ; V_{3 / 4}^{(k)}\right\|_{0}+\left\|u_{m} ; \gamma ; \beta ; 0 ; V_{3 / 4}^{(k)}\right\|_{2}+\left\|\psi_{m}^{(k)} ; \tilde{\gamma} ; \tilde{\beta} ; 0 ; V_{3 / 4}^{(k)} \cap\left(t=t_{k}\right)\right\|_{2+\alpha}\right) .
\end{gathered}
$$


Taking into account the interpolation inequalities and estimates of the norm of each addend in the expressions $F_{m}, \psi_{m}^{(k)}$, we obtain the inequalities

$$
\begin{aligned}
& E_{\delta} \leq\left(\varepsilon_{1}^{\alpha}(n+2)+\varepsilon^{2} n^{2}\right)\left\|u_{m} ; \gamma ; \beta ; 0 ; V_{3 / 4}^{(k)}\right\|_{2+\alpha}+c_{4}\left\|u_{m} ; V_{3 / 4}^{(k)}\right\|_{0}+ \\
& \quad+c_{5}\left(\left\|f_{m} ; \gamma ; \beta ; \mu_{0} ; V_{3 / 4}^{(k)}\right\|_{\alpha}+\left\|\psi_{m}^{(k)} ; \tilde{\gamma} ; \tilde{\beta} ; 0 ; V_{3 / 4}^{(k)} \cap\left(t=t_{k}\right)\right\|_{2+\alpha}\right),
\end{aligned}
$$

Where

$$
\begin{gathered}
\left\|\psi_{m}^{(0)} ; \tilde{\gamma} ; \tilde{\beta} ; 0 ; V_{3 / 4}^{(0)} \cap\left(t=t_{0}\right)\right\|_{2} \leq c_{6}\left\|\varphi_{0}^{(m)} ; \tilde{\gamma} ; \tilde{\beta} ; 0 ; \mathbb{R}^{n}\right\|_{2+\alpha}, \\
\left\|\psi_{m}^{(k)} ; \tilde{\gamma} ; \tilde{\beta} ; 0 ; V_{3 / 4}^{(k)} \cap\left(t=t_{k}\right)\right\|_{2+\alpha} \leq c_{7}\left(1+\left\|b_{k}^{(m)} ; \Pi \cap\left(t=t_{k}\right)\right\|_{0}\right) \times \\
\times\left(\left\|u_{m} ; \gamma ; \beta ; 0 ; \Pi^{(k-1)}\right\|_{2+\alpha}+\left\|\varphi_{k}^{(m)} ; \tilde{\gamma} ; \tilde{\beta} ; 0 ; \Pi^{(k)} \cap\left(t=t_{k}\right)\right\|_{2+\alpha}\right),
\end{gathered}
$$

for $k \in\{1,2, \ldots, N\}$.

Using inequalities (8), (12), (15), (26)-(28) and choosing $\varepsilon$ and $\varepsilon_{1}$ sufficiently small, we obtain the estimate (11).

Proof of Theorem 1. Since

$$
\begin{gathered}
\left\|f_{m} ; \gamma ; \beta ; \mu_{0} ; \Pi^{(k)}\right\|_{\alpha} \leq c\left\|f ; \gamma ; \beta ; \mu_{0} ; \Pi^{(k)}\right\|_{\alpha} \\
\left\|\varphi_{m}^{(k)} ; \tilde{\gamma} ; \tilde{\beta} ; 0 ; \Pi^{(k)} \cap\left(t=t_{k}\right)\right\|_{2+\alpha} \leq c\left\|\varphi_{k} ; \tilde{\gamma} ; \tilde{\beta} ; 0 ; \Pi^{(k)} \cap\left(t=t_{k}\right)\right\|_{2+\alpha},
\end{gathered}
$$

and estimate (11) holds, for the solution of problem (5)-(7) the estimate is true

$$
\left\|u_{m} ; \gamma ; \beta ; 0 ; \Pi\right\|_{2+\alpha} \leq B
$$

The right-hand side of inequality (29) does not depend on $m=\left(m_{1}, m_{2}\right)$. Moreover, the sequences

$$
\begin{aligned}
& \left\{U_{m}^{(0)}\right\}=\left\{u_{m}\right\} \\
& \left\{U_{m}^{(1)}\right\}=\left\{d_{1}\left(\gamma^{(1)}, t\right) d_{2}\left(\gamma^{(2)}-\beta_{i}^{(2)}, x\right) \partial_{x_{i}} u_{m}(t, x)\right\}, \\
& \left\{U_{m}^{(2)}\right\}=\left\{d_{1}\left(2 \gamma^{(1)} ; t\right) d_{2}\left(\gamma^{(2)}-\beta_{i}^{(2)}, x\right) d_{2}\left(\gamma^{(2)}-\beta_{j}^{(2)}, x\right) \partial_{x_{i}} \partial_{x_{j}} u_{m}(t, x)\right\}, \\
& \left\{U_{m}^{(3)}\right\}=\left\{d_{1}\left(2 \gamma^{(1)} ; t\right) d_{2}\left(2 \gamma^{(2)}, x\right) \partial_{t} u_{m}(t, x)\right\}
\end{aligned}
$$

are uniformly bounded and equicontinuous in the domain $\bar{Q}^{(k)}$. According to the Arzela theorem, there exist subsequences $\left\{U_{\tilde{m}_{k}}^{(\nu)}\right\}$, uniformly convergent in $\bar{Q}^{(k)}$ to $\left\{U_{0}^{(\nu)}\right\}, \nu \in$ $\{0,1,2,3\}$. Since $Q^{(k)}$ is an arbitrary domain, $\bar{Q}^{(k)} \subset \Pi^{(k)}$, then passing to the limit for $m_{1 k} \rightarrow \infty, m_{2 k} \rightarrow \infty$ in problem (5)-(7), we find that $u(t, x)=U_{0}^{(0)}$ is the unique solution of problem (1)-(3) in the space $H^{2+\alpha}(\gamma ; \beta ; 0 ; \Pi)$ and its estimate (4) holds.

\section{REFERENCES}

1. Perestyuk N.A., Plotnikov N.A., Samoilenko A.M., Skripnik N.V., Differential Equations with Impulse Effects, Berlin: Multivalued Right-hand Sides with Discontinuities - Walter de Gruyter Co, 2011. 408 p.

2. Samoilenko A.M., Perestyuk N.A. Impulsive Differential Equations. - Singapore: World Scientific, 1995. - 462 p. 
3. Boinov D.D., Simeonov P.S. Systems with impulse effects: Stability theory and applications. - New York etc.: John Wiley\&Sons, 1989. - 225 p.

4. Perestyk N.A., Tkach A.B. Periodic solutions of a weakly linear system of partial differential equations with impulse action// Ukr. Mat. Zh. - 1997. - №4.(49) - P. 601-605.

5. Asanova A.T. On a nonlocal boundary value problem for systems of hyperbolic equations with impulse actions// Ukr. Mat. Zh. - 2013. - №3.(65) - P. 315-328.

6. Pykal'skii I.D., Isaryuk I.M. The boundary-value problem with impulse conditions and degenerations for parabolic equations// Ukr. Mat. Zh. - 2015. - №10.(67) - P. 1399-1408.

7. Pykal'skii I.D. The boundary value problem for parabolic equations with impulse conditions and degenerations// Mathematical methods and physicomechanical fields - 2015. - №2.(58) - P. 55-63.

8. Isaryuk I.M., Pukalskyi I.D. The boundary value problems for parabolic equations with a nonlocal condition and degenerations// Journal of Mathematical Sciences. - 2015. - №1.(207) - P. 26-38.

9. Pukalskyi I.D. Isaryuk I.M. Nonlocal parabolic boundary value problems with singularities// Journal of Mathematical Sciences. - 2015. - №3.(208) - P. 327-343.

10. Friedman A. Partial differential equations of parabolic type. - M.: World, 1968. - 427p.

11. Ladyzhenskaya O. A., Solonnikov V. A., Ural'tseva N. N. Linear and quasilinear equations of parabolic type. - M.: Science, 1967. - 736p.

12. Pukalskyi I.D. The boundary value problems for unevenly parabolic and elliptic equations with degeneration and singularities. - Chernivtsi: Ruta, 2008. - 253p.

Yuriy Fedkovych Chernivtsi National University

Chernivtsi, Ukraine

bohdanjaschan94@gmail.com 